\title{
The Course Construction and Reform of Python Programming in the Background of Artificial Intelligence
}

\author{
Yongcheng $\mathrm{Wu}$ \\ Computer Engineering School \\ Jingchu University of Technology \\ Jingmen, China \\ wuyongcheng11@126.com
}

\begin{abstract}
Nowadays artificial intelligence is profoundly changing the world. Since Python is the most popular Artificial intelligence programming language at present, teaching of Python is becoming more and more critical in colleges. In this paper, after discussing the significance and advantages of Python language, we put forward that before the teaching task we firstly should set the goal of Python teaching according to the condition of our local applied college. Then specific teaching syllabus and teaching contents are formulated for Python teaching. Finally, the methods of teaching reform and evaluation of the course of Python Language are also discussed in this paper.
\end{abstract}

Keywords-course construction; Python programming; teaching reform; artificial intelligence

\section{INTRODUCTION}

The rapid development of artificial intelligence (AI) is profoundly changing human life and the world. In 2017, China issued a landmark AI development plan, New Generation AI Development Planning, which provides strategic support for the development of AI in China [1]. In 2018, China's Ministry of Education formulated the Action Plan for Artificial Intelligence Innovation in Colleges, which explicitly requires that AI must be included in the teaching contents of Fundamentals of Computers [2].

With the rapid development of AI and Big Data technology, Python has become the most popular AI programming language in the world since 2017 [3].

In 2017, the Ministry of Education's College Computer Courses Teaching Guiding Committee issued Basic Requirements for College Computer Basic Courses Teaching, which recommended that Python language should be the preferred teaching language for programming courses [4].

In October 2017, China's National Education Examinations Authority (NEEA) stipulated that the subject of Python Language Programming should be included into the National Computer Rank Examination (NCRE), which was implemented in March 2018 and the first time examination of Python of NCRE was held in September 2018.

Compared with other languages, Python language has higher teaching value. Stanford University in the United States, for example, has offered Python language programming courses since 2009, adding about five courses every year. By 2015, it has offered 22 courses related to Python language, and replaced the previous Java language and C language courses in some majors. More than 70 of the top 100 universities in the United States had offered Python language courses from 2014 to 2016 [5].

Most colleges and universities use traditional $\mathrm{C} / \mathrm{C}++$ or Java as their students' programming introductory language. Since $\mathrm{C} / \mathrm{C}++$ or Java language adopt more complex grammar rules to improve the performance of program, it is easy to make students fall into the details of tedious grammar rules and ignore the essence of programming, which will reduce students' interest in programming, especially for local application-oriented undergraduate colleges [6].

Python not only provides strong support for AI, but also is called the programming language closest to human natural language, which is convenient for people to learn and use. It attaches much more importance to the readability and conciseness of the program, abstracts a lot of contents, lets developers focus on business logic rather than grammar details, and can complete more powerful functions by using as little code as possible. It can not only cultivate students' interest in learning program design, but also improve students' application ability and practical project ability.

\section{The Significance of Python LANGUAGE}

Python language is easy to learn, easy to use and has rich open source libraries, which will bring students a new experience of programming, and would change the situation in which students want to learn programming language well, but objectively cannot learn it well. For local application-oriented undergraduate colleges like our university, the overall quality of students is not high. Whether computer or non-computer students, in the past they were taught to learn programming basis in $\mathrm{C} / \mathrm{C}++$ or Java language. Since $\mathrm{C} / \mathrm{C}++$ or Java is rather difficult, the interest of students in learning were little and the effect of learning were very poor. If we use Python to learn the basis of programming, we believe that it will greatly enhance students' interest in learning, thereby improving the quality of teaching. 
Python is the closest programming language to natural language at present. Its lightweight grammar and high-level language representation are more conducive to cultivating students' ability to solve practical problems by computer [7].

Figure.1, Figure.2, Figure.3 show the program used to calculate the sum of two numbers in $C$ language, Java language and Python language respectively.

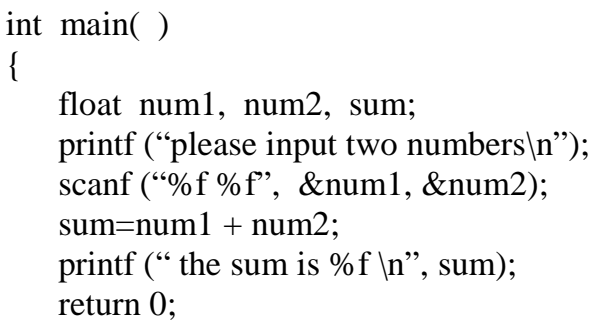

Fig. 1. Calculating the sum of two numbers in $\mathrm{C}$ language

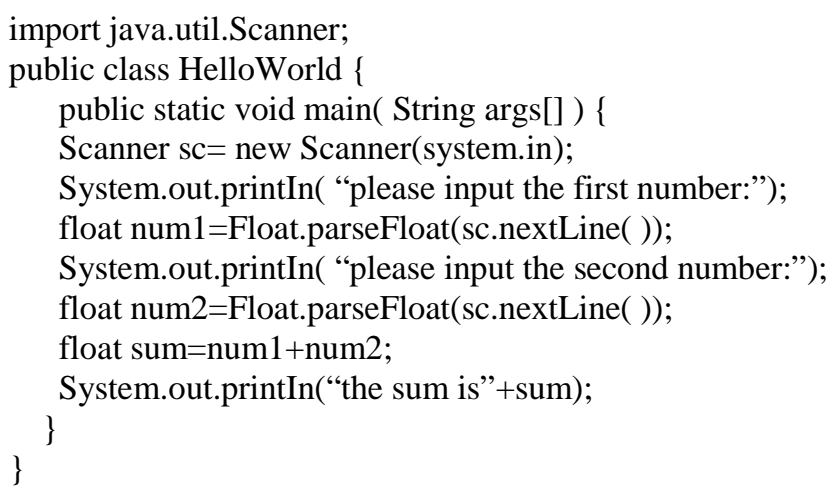

Fig. 2. Calculating the sum of two numbers in Java language

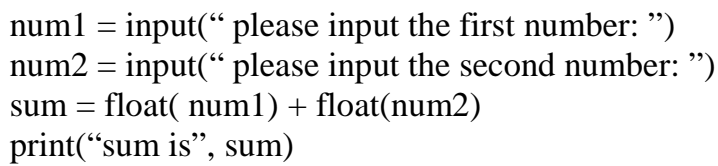

Fig. 3. Calculating the sum of two numbers in Python language

From the Fig.1, Fig.2, Fig.3, we can see that for the same question, Python only needs 4 statements, while $\mathrm{C}$ needs 6 statements and Java needs 10 statements. It is also clearly shown that Python is simpler, more readable, and more consistent than $\mathrm{C} / \mathrm{C}++$ and Java.

Finally, using Python's strong support for AI and machine learning, we can promote the spread and application of AI technology, which is particularly important under the background of AI.

\section{PREPARATIONS FOR Python LANGUAGE TEACHING}

In order to teach the course of Python Language smoothly and productively, we must firstly do the following preparations:

\section{A. Setting the Main Teaching Objective}

Since the level and quality of our university is local applied undergraduate college, we should formulate and revise the teaching objectives. For basic programming courses, our school used to use $\mathrm{C} / \mathrm{C}++$ language as the description language. These languages generally adopt more complex grammar rules in order to take into account performance. This would lead to too much emphasis on grammar teaching, which tends to trap students into tedious grammar formats and other details while ignoring the essence of programming. It would reduce students' interest in learning programming and is not conducive to the shaping and cultivation of students' programming ability. The grammar of Python is simple and has rich and powerful third-party class libraries. Python can be used as a basic programming description language to develop various applications efficiently. Therefore its main teaching objective should be to cultivate students' ability to solve practical problems by using computers, rather than the knowledge of Python language grammar itself.

\section{B. Formulating Syllabuses}

A syllabus is an outline or summary of the subjects to be covered in a course, and it is also the guide for the task of course teaching. For students of computer and non-computer majors, different syllabuses should be formulated firstly.

The teaching content of Python Language can be divided into two parts: the basic part and the application part.

Our university belongs to local applied undergraduate colleges, and the overall quality of students is not very high. So for the basic part of Python Language, just like the basic words and grammar rules of English Language, basic statements and grammar rules are enough. Otherwise, it is prone to reduce students' interest in learning, leading to the situation in which students want to learn programming well subjectively but could not learn well objectively.

The basic part mainly includes: Python basic grammar rules, common statements, basic data structures, basic functions and Python object-oriented programming foundation.

Learning the application part is the real purpose of learning this course. Only mastering this part can students really program to solve some practical problems, which will stimulate the enthusiasm of learning programming of students

The application part includes three basic modules which are the most closely related to AI: the basic usage of SQLite database, the basic application methods of Python+Pandas data processing, and the basic usage of Python+sklearn machine learning library.

- SQLite: SQLite is a very lightweight database. Therefore, embedded software for computer, mobile phone, camera, household electronic equipment and other devices is a very good choice. SQLite provides a 
simple and effective way to process data, rather than using memory variables for data processing. For example, if you are developing a program and have some records to calculate it. Then, you can create a SQLite database and insert records into it; query, select records, and performs the required calculations directly.

- Python+Pandas: Pandas is a tool based on NumPy, which is created to solve data analysis tasks. Pandas incorporates a large number of libraries and some standard data models, providing the tools needed to operate large data sets efficiently. Pandas provide a large number of functions and methods that enable us to process data quickly and conveniently. It is one of the important factors that make Python a powerful and efficient data analysis environment.

- Python+sklearn: sklearn is an important machine learning library that is widely used. It encapsulates a large number of machine learning algorithms, such as classification, clustering, regression, dimensionality reduction, etc. It also includes three modules: supervised learning, unsupervised learning and data transformation. In the course of Python teaching, the clustering method is taken as an example. K-means and DBSCAN are very simple clustering algorithms. Their principles are very clear and can be used as teaching cases.

Preliminary design of the proportion of each part in the total teaching hours of Python Language is: the basic part and the application part account for $50 \%$ respectively. The proportion of three modules in the application part is $15 \%$ (SQLite), 15\% (Python+Pandas ) and 20\% (Python+sklearns) respectively.

Table 1 shows the Preliminary time distribution of the course of Python Language.

TABLE I. PRELIMINARY TIME DISTRIBUTION

\begin{tabular}{|l|l|c|}
\hline \multicolumn{2}{|c|}{ Teaching Contents } & $\begin{array}{c}\text { Teaching Time } \\
\text { Distribution }\end{array}$ \\
\hline \multirow{5}{*}{$\begin{array}{l}\text { Basic part } \\
\text { (50\%) }\end{array}$} & $\begin{array}{l}\text { Variables, Data Types, } \\
\text { Expressions }\end{array}$ & $5 \%$ \\
\cline { 2 - 3 } & $\begin{array}{l}\text { if Statement and Looping } \\
\text { Statements }\end{array}$ & $5 \%$ \\
\cline { 2 - 3 } & Character string & $5 \%$ \\
\cline { 2 - 3 } & List, Tuple, Dictionary & $5 \%$ \\
\cline { 2 - 3 } & Functions & $5 \%$ \\
\cline { 2 - 3 } & File System & $5 \%$ \\
\cline { 2 - 3 } & $\begin{array}{l}\text { Object-oriented } \\
\text { Programming }\end{array}$ & $20 \%$ \\
\hline \multirow{4}{*}{$\begin{array}{l}\text { Application } \\
\text { part(50\%) }\end{array}$} & SQLite & $15 \%$ \\
\cline { 2 - 3 } & Python+Pandas & $15 \%$ \\
\cline { 2 - 3 } & Python+sklearn & $100 \%$ \\
\hline \multirow{2}{*}{ Total } & & \\
\hline
\end{tabular}

\section{Selecting Textbook and Preparing lessons}

According to the characteristics of local applied undergraduate Colleges just as our university, we should teach students in accordance with their actual level and ability, select appropriate teaching materials, and prepare teaching plans and courseware in accordance with the requirements of the syllabus. Especially in the selection of teaching examples, students should be allowed to learning the lessons through practical using. The pace of learning should not be too fast. It should move forward step by step. All the examples and projects should have the practical application background, especially the application background of artificial intelligence, which is very useful to stimulate students' interest in learning, and cultivate students' ability to solve practical problems.

\section{REFORM OF TEACHING MEthod AND EVALUATION}

\section{A. Reform of Teaching Method}

The focus of the teaching goal of the courses of Programming is to cultivate students' ability to solve practical problems by using computers. The traditional teaching process of this kind of course is "theory + practice". That is to teach theoretical knowledge in class first and then practice through practical operation.

The core of the new method is to let students learn in practice, learn grammar in examples, and improve the ability of programming and problem solving in practice.

To explore the new teaching method suitable for Python Programming, the key is to improve students' interest in learning, to teach less grammar, to learn computer programming language in practice, rather than to learn language for the sake of language itself.

Since the Python interactive environment is a real language editing and executing environment, teachers can design some traps at any time to enhance students' integration in the teaching process, activate the classroom atmosphere, and stimulate students' enthusiasm for thinking.

\section{B. Reform of Evaluation}

The aim of reforming the assessment model and evaluation method is to encourage students to attach more importance to innovation and practice, and encourage teamwork and sharing. The proportion of "comprehensive design experiment" is to be increased. It is also necessary to associate experiment themes with AI and machine learning. Students should be encouraged to bring their imagination and innovation into full play. The assessment score will be composed of normal performance (20\%), comprehensive design experiment report and demonstration (40\%) and final examination (40\%).

Fig.4 illustrates how to evaluate the performance of teaching of the course of Python Language.

\section{CONCLUSION}

In this paper, we analyze the importance of $\mathrm{AI}$ and the nececity and urgency of offering the course of Python Language in colleges. After that, we put forward the main 
contents of Python Language that should be mastered by the students, which consists of basic part and application part. The basic part is mainly composed of the basic statements and data structures of Python. The application part consists of three modules that is closely related to AI: SQLite, Pandas and sklearn. We also provide some suggestions of how to teach the students productively and how to evaluate the students' performance precisely.

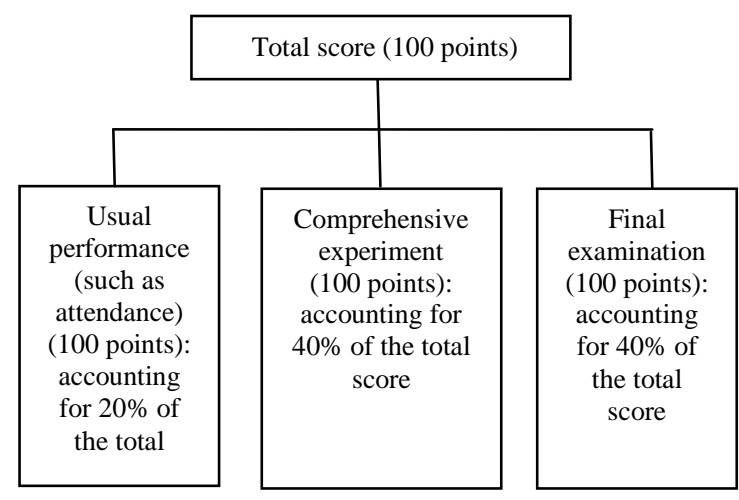

Fig. 4. Evaluation model of the course of Python Language

\section{REFERENCES}

[1] New Generation Development Planning of Artificial Intelligence [Z] Central Government file GuoFa [2017] 35. http://www.gov.cn/zhengce/content/201707/20/content 5211996.htm. (In Chinese)

[2] Action Plan for Artificial Intelligence Innovation in Colleges. Ministry of Education file JiaoJi [2018] 3. http://www.cac.gov.cn/201804/11/c_1122663790.htm. (In Chinese)

[3] Y. Liu, X. Lai, P. Li, "Research on the Python case teaching in the background of artificial intelligence”, in Computer Era, 2018(4):88-92. (In Chinese)

[4] D. Wei, X. Wang, Y. Wang, Y, Chen, S. Wang, "Construction and Teaching Practice of Programming General Course Based on Python”, in Computer Education, 2019(2): 69-73. (In Chinese)

[5] T. Song, T. Huang, X. Li, "Python Language: Ideal Choice for Teaching Reform of Programming Course”, in China University Teaching, 2016(2): 42-47. (In Chinese)

[6] R. Yang, "Teaching Reform and Practice of Basic Python Language Programming”, in Journal of Higher Education, 2019(3): 135-137. In Chinese

[7] Hong. Jiang, Q.S. Yu, Python Programming and Computer Fundamentals. Beijing, Tsinghua University Press, 2017. (In Chinese) 